\title{
Chronic Pelvic Pain Syndrome in a Rural Area: Are We Paying Attention?
}

Omar S. Akhtar 1,2

1. Urology, Hakim Sanaullah Specialist Hospital, Sopore, IND 2. Urology, Government Medical College, Srinagar, IND

Corresponding author: Omar S. Akhtar, omarakhtar@hsshcc.org

\begin{abstract}
Introduction

Chronic prostatitis/chronic pelvic pain syndrome (CP/CPPS) is a chronic, debilitating illness that mostly affects males under the age of 50 years. It is associated with myriad presentations. The National Institutes of Health-Chronic Prostatitis Symptom Index (NIH-CPSI) score was developed to measure the impact of the disease and assess the treatment outcomes. Additionally, the UPOINT [urinary (U), psychosocial (P), organspecific (O), infection (I), neurologic/systemic $(\mathrm{N})$, and tenderness of pelvic floor skeletal muscles $(\mathrm{T})$ ] classification system has been developed to enable a 'phenotypic approach' to the treatment.
\end{abstract}

\section{Objective}

The objective of this study was to determine the NIH-CPSI scores as well as the positive subdomain numbers and distribution of the UPOINT classification in patients with CP/CPPS.

\section{Materials and methods}

A total of 100 consecutive male patients presenting to a single centre with symptomatic CP/CPPS were included in this study.

\section{Results}

The mean age of the patients was 34.4 years. The average total NIH-CPSI score was 24.9. The average number of positive UPOINT domains was 2.28. The positive domains were urinary (90), psychosocial (60), organ-specific (43), infection (15), neurological (12), and tenderness (8).

\section{Discussion}

The NIH-CPSI scores and UPOINT subdomain scores compared favourably with other studies conducted in the region. The lower infection subdomain score as compared to other studies may be due to the widespread use of antibiotics among patients in the region prior to presenting to a urologist.

Review began 02/11/2021 Review ended 02/16/2021 Published 02/23/2021

\section{○ Copyright 2021}

Akhtar. This is an open access article distributed under the terms of the Creative Commons Attribution License CC-BY 4.0., which permits unrestricted use, distribution, and reproduction in any medium, provided the original author and source are credited.

\section{Conclusion}

The use of the UPOINT classification to guide treatment is feasible even in a rural setting, such as in Kashmir.

\footnotetext{
Categories: Pain Management, Urology, Public Health

Keywords: chronic pelvic pain syndrome, lower urinary tract synptoms, prostatitis, chronic prostatitis
}

\section{Introduction}

Prostatitis-like symptoms are a common reason for outpatient visits to urologists among male patients under the age of 50 years, with a prevalence of 2-9\% [1]. The National Institutes of Health (NIH) has classified prostatitis into four categories - type I: acute bacterial prostatitis; type II: chronic bacterial prostatitis; type III: chronic nonbacterial prostatitis or chronic pelvic pain syndrome (CNP/CPPS); and type IV: asymptomatic inflammatory prostatitis [2]. Type III is subclassified into IIIA (inflammatory) and IIIB (non-inflammatory), based on the presence of leucocytes in expressed prostatic secretions, urine specimen after the prostatic massage, or semen [2]. Chronic prostatitis type III or chronic pelvic pain syndrome (CP/CPPS) has a varied presentation but is most often characterised by genital pain, urinary problems, erectile and ejaculatory dysfunction, and psychological problems [3]. Erectile dysfunction (ED), which is the inability to develop and/or maintain an erection, is the most common sexual dysfunction reported along with CP/CPPS and has been reported in 46-92\% of patients with CP/CPPS [4]. CP/CPPS is associated with myriad presentations, and different treatment methods, which necessitated a 'phenotypic approach' first advocated by Shoskes et al. in 2009 for the diagnosis and treatment of CP/CPPS. They proposed a 'UPOINT' classification or 'Snowflake' model, consisting of six subdomains of urinary, psychosocial, organ-specific, infection, neurological or systemic abnormalities, and muscle or skeletal tenderness (UPOINT) [5]. The UPOINT-directed treatment 
The study presented here is a prospective study of the use of the NIH-Chronic Prostatitis Symptom Index (NIH-CPSI) score and the UPOINT classification in a predominantly rural population of patients presenting to a single centre with features suggestive of CP/CPPS.

\section{Materials And Methods}

A total of 100 consecutive patients who met the criteria of CP/CPPS were examined by a single urologist in the urology outpatient department at the Hakim Sanaullah Specialist Hospital and Cancer Centre in Sopore, Kashmir, India, from April 2018 to December 2020. After a thorough history and physical examination, a two-glass test for urinary tract infection was performed to localise the infection to the prostate. This has been shown to be equivalent to the four-glass test [7]. All patients were evaluated according to the NIH-CPSI scores, with subscores determined based on pain (0-21 points), quality of life (QoL, 0-12 points), and urinary (0-10 points) scores (total score range: $0-43$ points). Patients were classified into three domains: severe dysfunction ( $>29$ points), moderate dysfunction (16-29 points), and mild dysfunction (0-15 points) according to their degree of symptoms as per the NIH-CPSI score. This method has been used previously in a study conducted in Turkey by Arda et al. [8]. The UPOINT framework was used to evaluate patients according to the relevant criteria. In all patients, at least one UPOINT subdomain positivity was present. Patients were specifically asked about their sexual history and the findings were recorded. All patients underwent a urinalysis, routine blood tests, and an ultrasound of the kidney, ureter, bladder, and prostate with the measurement of post-void urine volume and a pre- and post-prostatic massage urine culture.

The urinary subdomain was considered positive if there was high post-void residual volume (>100 ml, as measured by ultrasound), bothersome nocturia, urgency, and frequency of urination, or a CPSI urine subdomain score of $\geqslant 4$. The psychosocial subdomain was determined to be positive if the patients stated that they were depressed and felt hopeless due to the disease. Organ-specific subdomain was considered to be positive if there was prostate tenderness upon rectal palpation, haematospermia, or sign of intense prostatic calcifications on ultrasound. The infection subdomain was deemed positive in patients other than in category I and II CP if growth was seen on urine culture on the second sample of the two-glass test. Neurological or systemic subdomain positivity was associated with pain outside of the pelvis or newly diagnosed irritable bowel syndrome, or chronic fatigue syndrome. Finally, the tenderness subdomain was considered positive if there was palpable muscle spasm or abdominopelvic trigger points. The phenotypic approach to diagnose patients was followed. Patients were treated using the multimodal treatment pathway as advocated by Shoskes et al. [6], as summarised in Table 1 . 


\section{Cureus}

\begin{tabular}{|c|c|}
\hline Domain & Clinical description \\
\hline \multirow{4}{*}{ Urinary } & CPSI score of $>4$ \\
\hline & Bothersome urgency, frequency, nocturia \\
\hline & A flow rate of < $15 \mathrm{ml} / \mathrm{second}$, or obstructed pattern \\
\hline & Post-void residue of $>100 \mathrm{ml}$ \\
\hline \multirow{2}{*}{ Psychosocial } & Depression \\
\hline & Poor coping or maladaptive behavior, catastrophizing \\
\hline \multirow{4}{*}{ Organ-specific } & Prostate tenderness \\
\hline & Haematospermia \\
\hline & Leucocytosis in the prostatic secretion \\
\hline & Extensive prostatic calcification \\
\hline \multirow{3}{*}{ Infection } & $\begin{array}{l}\text { Excluded patients with evidence of acute or chronic bacterial prostatitis (recurrent - located to the } \\
\text { prostate in repeated specimens) }\end{array}$ \\
\hline & Gram-negative bacilli \\
\hline & Documented response to antibacterial therapy \\
\hline \multirow{3}{*}{$\begin{array}{l}\text { Neurologic/systemic } \\
\text { conditions }\end{array}$} & Pain beyond pelvis \\
\hline & Irritable bowel syndrome \\
\hline & Chronic fatigue syndrome \\
\hline Tenderness & $\begin{array}{l}\text { Palpable tenderness and/or painful muscle spasm or trigger points in the perineum or pelvic floor or } \\
\text { pelvic sidewalls during DRE }\end{array}$ \\
\hline
\end{tabular}

TABLE 1: Subdomain positivity based on Shoskes et al.*

*[6]

CPSI: Chronic Prostatitis Symptom Index; DRE: digital rectal examination

The exclusion criteria were as follows: patients with acute bacterial prostatitis, chronic bacterial prostatitis, history of genitourinary cancer or genitourinary tuberculosis, urethral stricture, history of recent endourological procedure, or a diagnosis of neurogenic bladder.

\section{Statistical analysis}

Data were analysed statistically using the available software in Microsoft Excel (Office 365, Microsoft Corporation, Redmond, WA), and Google Drive (Google, Alphabet Inc., Mountain View, CA). The UPOINT and NIH-CPSI scores of patients were analysed. Data were presented as means and standard deviations. A pvalue of $<0.05$ was considered statistically significant.

\section{Results}

A total of 100 consecutive patients were enrolled in the study. Demographic data of the subjects are presented in Table 2 . 


\section{Cureus}

\section{Variables}

Mean age, years (range, SD)

Residence type, n (\%)

Profession, n (\%)

Average duration of symptoms, months (range, SD)

\section{NIH-CPSI scores}

Total, average (range, SD)

Pain, average (range, SD)

Voiding, average (range, SD)

QoL, average (range, SD)

IPSS, average (range, SD)

Pain severity, $\mathrm{n}(\%)$

Mild

Moderate

Severe
Values

$34.37(21-60,8.55)$

Rural: 98/100 (98\%); urban 2/100 (2\%)

Salaried: 11/100 (11\%); daily-wage earner: 34/100 (34\%); self-employed: 10/100 (10\%); farmer/orchardist: 45/100 (45\%)

$8(3-24,5.01)$

$24.9(14-34,5.06)$

$11.4(4-18,3.49)$

$6.3(2-8,1.85)$

$7.2(4-11,2.14)$

$9.4(4-26,4.74)$

$10(10 \%)$

$66(66 \%)$

$24(24 \%)$

\section{TABLE 2: Demographic profile of patients}

SD: standard deviation; NIH-CPSI: National Institutes of Health-Chronic Prostatitis Symptom Index; QoL: quality of life; IPSS: International Prostate Symptom Score

The NIH-CPSI and UPOINT subdomain data of these patients were analysed. The average total NIH-CPSI score was 24.9 (range: 14-34, SD: 5.06) and the average positive UPOINT subdomain number was 2.28 (Table 3, Figure 1). Positive subdomains were determined as follows - urinary: 90 (90\%); psychosocial: 60 (60\%); organ-specific: 43 (43\%); infection: 15 (15\%); neurological or systemic: 12 (12\%); and tenderness: 8 (8\%). For validating the UPOINT and NIH-CPSI scores, we calculated the correlation between positive UPOINT subdomain number and NIH-CPSI total score and subdomain pain (0-21 points), QoL (0-12 points), and urinary (0-10 points) scores in the patient population (Figure 2). A correlation was seen between UPOINT subdomain positivity and NIH-CSPI total score. When the subdomains urinary, pain, and QoL were considered separately, a correlation with UPOINT subdomain positivity was observed. It was also observed that as the UPOINT subdomain number positivity increased, there was a gradual increase in the NIH-CPSI score.

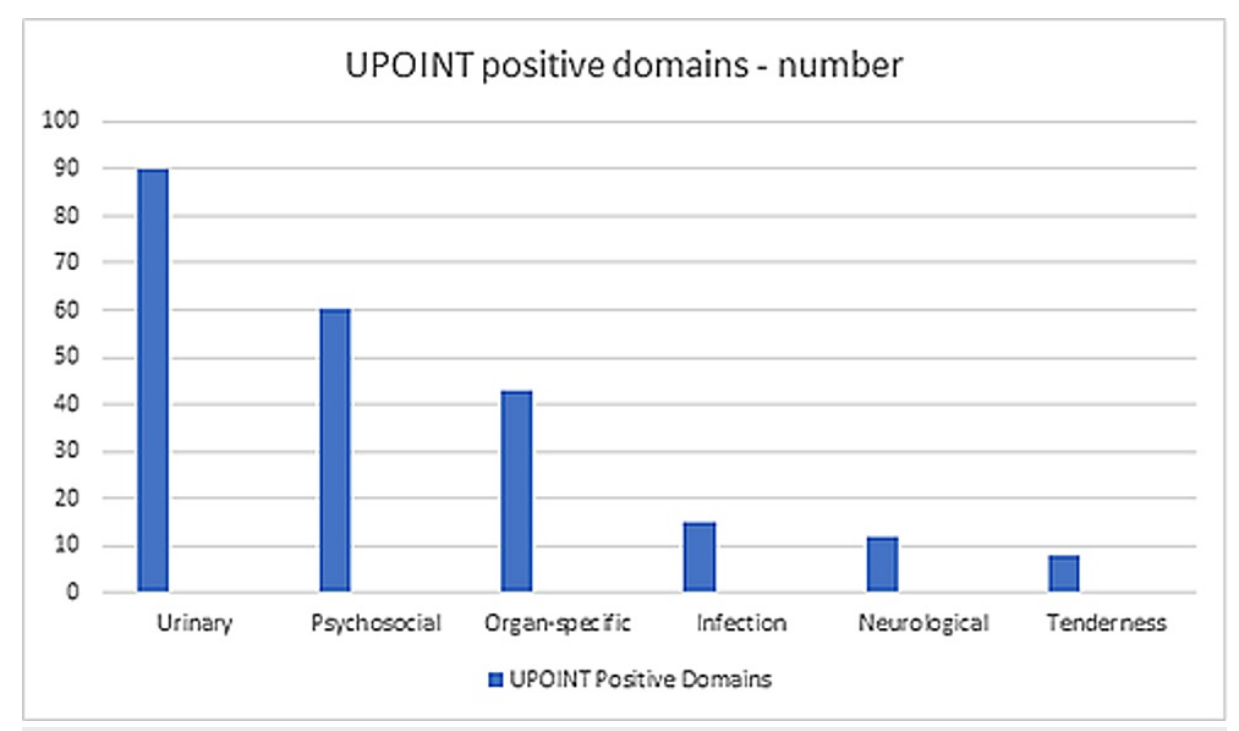




\section{Cureus}

\section{FIGURE 1: Positive domains among patients}

UPOINT: urinary $(\mathrm{U})$, psychosocial $(\mathrm{P})$, organ-specific $(\mathrm{O})$, infection $(\mathrm{I})$, neurologic/systemic $(\mathrm{N})$, and tenderness of pelvic floor skeletal muscles $(T)$

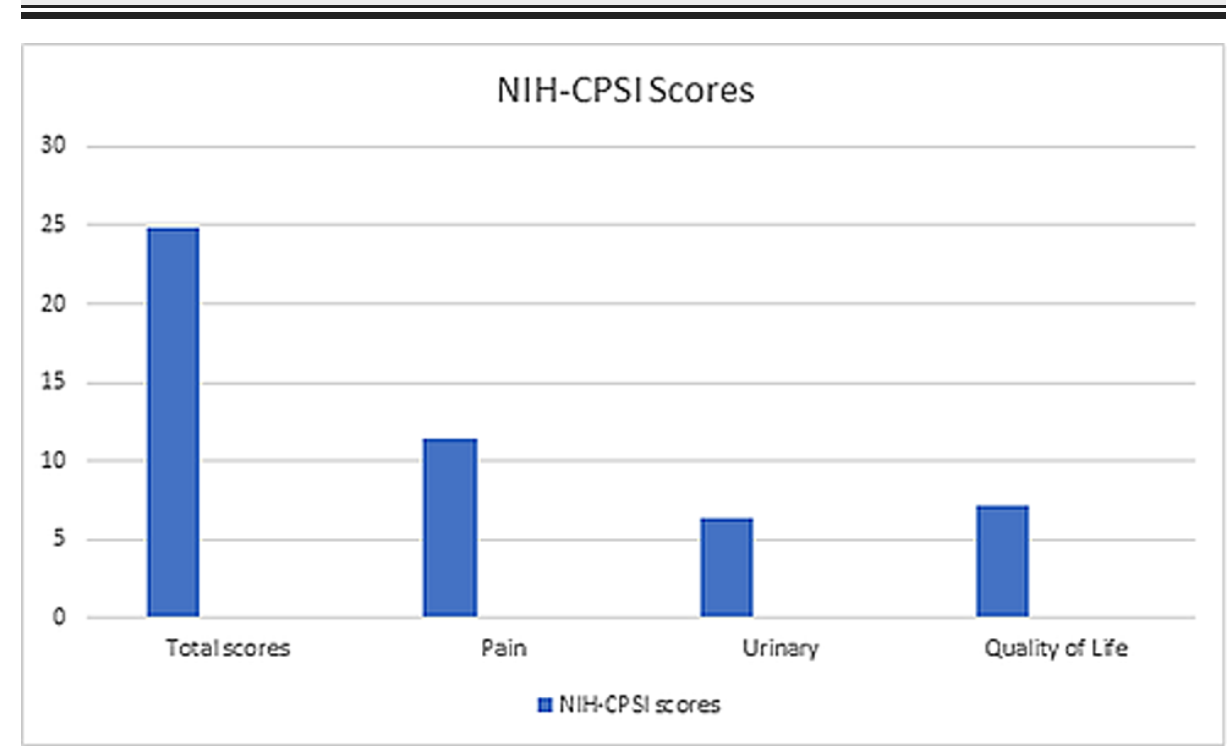

FIGURE 2: NIH-CPSI scores

NIH-CPSI: National Institutes of Health-Chronic Prostatitis Symptom Index

Domain (percentage positive)

Urinary

Psychosocial

Organ-specific

Infection

Neurological/systemic

Skeletal muscle tenderness
Number (\%) of positive scores

$90(90 \%)$

$60(60 \%)$

$43(43 \%)$

$15(15 \%)$

$12(12 \%)$

$8(8 \%)$

\section{TABLE 3: UPOINT positive domains}

UPOINT: urinary $(\mathrm{U})$, psychosocial $(\mathrm{P})$, organ-specific $(\mathrm{O})$, infection $(\mathrm{I})$, neurologic/systemic $(\mathrm{N})$, and tenderness of pelvic floor skeletal muscles $(\mathrm{T})$

The total number of patients with CP/CPPS constituted 7\% of the total urology outpatient visits during the study period (Table 4). 


\title{
Cureus
}

Urology outpatient department visits

Total

CP/CPPS diagnosis
1,418

$100(7 \%)$

\section{TABLE 4: CP/CPPS patients out of total urology outpatient visits}

\author{
CP/CPPS: chronic prostatitis/chronic pelvic pain syndrome
}

\section{Discussion}

CP/CPPS remains a common condition that impacts many males under the age of 50 years [9]. Wagenlehner et al. have shown that a classification-directed approach could improve the treatment outcomes of this disease since it has a varied presentation [10]. Shoskes et al. came up with the UPOINT classification, which is a phenotypic approach to CP/CPPS, in the same year [5]. However, an effective treatment method for $\mathrm{CP} / \mathrm{CPPS}$ remains elusive, and some studies have yielded negative or conflicting results [11-13]. Many patients have reported spending a considerable amount of time for clinic visits and money on medications, without achieving any resolution of their symptoms, which is an important consideration in our study population, a significant proportion of whom are rural and daily-wage earners [14]. Multimodal therapy based on the patient's UPOINT phenotype has been reported to achieve impressive results [8].

Our study showed a correlation between NIH-CPSI scores and the number of positive domains in the UPOINT score, which was in line with the findings of Shoskes et al. [5]. The number of positive UPOINT domains correlated with the symptom severity as measured by NIH-CPSI scores. There was an increase in the NIH-CPSI total score, pain subscore, urinary subscore, and Qol subscore as the number of positive UPOINT domains increased.

In this study, the average duration of symptoms among patients before presentation to the urologist was eight months. This observation validates the hypothesis that ongoing local inflammation and coincident tissue injury can cause local muscle spasms, with the central nervous system and peripheral nervous system alterations, and can cause psychosocial changes that can maintain the symptoms for many months after the initial insult has passed [13].

The mean age of the patients in our study was 34.4 years. The prevalence and the distribution of positive UPOINT domains and the NIH-CPSI scores in our patients were similar to those described in the Chinese $[14,15]$ and Turkish [8] studies on CP/CPPS. The similarity of results between different patient populations in different countries and health systems underlines the usefulness of the UPOINT phenotype system. Although the study sample was small, the results of our study among Kashmiri patients confirm the utility of the UPOINT algorithm for classifying CP/CPPS.

The number of patients who presented with positive Infection subdomain scores $(15,15 \%)$ was less compared to other studies $[8,14,15]$. This is probably a result of the widespread use of antibiotics in the region. It is plausible that many patients who had mild urinary tract or prostatic infections had already been treated for their infections, and had become culture-negative before presenting to the study centre.

Our study was not designed to evaluate the results of multimodal therapy, as has been done in other studies [15]. However, we were able to use the UPOINT phenotype to direct multimodal treatment successfully, as opposed to monotherapy previously.

The limitations of this study include the smaller sample size compared to other studies and the fact that this was a single-centre study. This study was conducted in a rural centre. Many patients had been seen and cared for by other physicians prior to their presentation to our hospital. Hence, many patients with less severe symptoms may have been left out. The study was not designed to include treatment outcomes, and we did not include a sexual-dysfunction domain in our analysis.

\section{Conclusions}

Based on our findings, the UPOINT classification system and the NIH-CPSI score can be effectively used in a predominantly rural population to evaluate and direct multimodal treatment for CP/CPPS. Multi-centric studies are required to further analyse the UPOINT system and NIH-CPSI scores, and to assess multimodal treatment outcomes and the relationship of CP/CPPS with sexual dysfunction within this population.

\section{Additional Information}




\section{Disclosures}

Human subjects: Consent was obtained or waived by all participants in this study. Animal subjects: All authors have confirmed that this study did not involve animal subjects or tissue. Conflicts of interest: In compliance with the ICMJE uniform disclosure form, all authors declare the following: Payment/services info: All authors have declared that no financial support was received from any organization for the submitted work. Financial relationships: All authors have declared that they have no financial relationships at present or within the previous three years with any organizations that might have an interest in the submitted work. Other relationships: All authors have declared that there are no other relationships or activities that could appear to have influenced the submitted work.

\section{References}

1. Krieger JN, Lee SW, Jeon J, Cheah PY, Liong ML, Riley DE: Epidemiology of prostatitis. Int J Antimicrob Agents. 2008, 31:S85-90. 10.1016/j.ijantimicag.2007.08.028

2. Propert KJ, McNaughton-Collins M, Leiby BE, O'Leary MP, Kusek JW, Litwin MS; Chronic Prostatitis Collaborative Research Network: A prospective study of symptoms and quality of life in men with chronic prostatitis/chronic pelvic pain syndrome: the National Institutes of Health Chronic Prostatitis Cohort study. J Urol. 2006, 175:619-23. 10.1016/S0022-5347(05)00233-8

3. Nickel JC, Nyberg LM, Hennenfent M: Research guidelines for chronic prostatitis: consensus report from the first National Institutes of Health International Prostatitis Collaborative Network. Urology. 1999, 54:229-33. 10.1016/s0090-4295(99)00205-8

4. Magri V, Boltri M, Cai T, et al.: Multidisciplinary approach to prostatitis. Arch Ital Urol Androl. 2019, 90:227248. 10.4081/aiua.2018.4.227

5. Shoskes DA, Nickel JC, Dolinga R, Prots D: Clinical phenotyping of patients with chronic prostatitis/chronic pelvic pain syndrome and correlation with symptom severity. Urology. 2009, 73:538-42. 10.1016/j.urology.2008.09.074

6. Shoskes DA, Nickel JC, Kattan MW: Phenotypically directed multimodal therapy for chronic prostatitis/chronic pelvic pain syndrome: a prospective study using UPOINT. Urology. 2010, 75:1249-53. 10.1016/j.urology.2010.01.021

7. Nickel JC: Prostatitis. Can Urol Assoc J. 2011, 5:306-15. 10.5489/cuaj.11211

8. Arda E, Cakiroglu B, Tas T, Ekici S, Uyanik BS: Use of the UPOINT classification in Turkish chronic prostatitis or chronic pelvic pain syndrome patients. Urology. 2016, 97:227-31. 10.1016/j.urology.2016.07.023

9. McNaughton Collins M, Pontari MA, O'Leary MP, et al.: Quality of life is impaired in men with chronic prostatitis: the Chronic Prostatitis Collaborative Research Network. J Gen Intern Med. 2001, 16:656-62. 10.1111/j.1525-1497.2001.01223.x

10. Wagenlehner FM, Schneider H, Ludwig M, Schnitker J, Brähler E, Weidner W: A pollen extract (Cernilton) in patients with inflammatory chronic prostatitis-chronic pelvic pain syndrome: a multicentre, randomised, prospective, double-blind, placebo-controlled phase 3 study. Eur Urol. 2009, 56:544-51. 10.1016/j.eururo.2009.05.046

11. Nickel JC, Krieger JN, McNaughton-Collins M, et al.: Alfuzosin and symptoms of chronic prostatitis-chronic pelvic pain syndrome. N Engl J Med. 2008, 359:2663-73. 10.1056/NEJMoa0803240

12. Mehik A, Alas P, Nickel JC, Sarpola A, Helström PJ: Alfuzosin treatment for chronic prostatitis/chronic pelvic pain syndrome: a prospective, randomized, double-blind, placebo-controlled, pilot study. Urology. 2003, 62:425-9. 10.1016/s0090-4295(03)00466-7

13. Pontari MA, Ruggieri MR: Mechanisms in prostatitis/chronic pelvic pain syndrome. J Urol. 2008, 179:S61-7. 10.1016/j.juro.2008.03.139

14. Zhao Z, Zhang J, He J, Zeng G: Clinical utility of the UPOINT phenotype system in Chinese males with chronic prostatitis/chronic pelvic pain syndrome (CP/CPPS): a prospective study. PLoS One. 2013, 8:e52044. 10.1371/journal.pone.0052044

15. Guan X, Zhao C, Ou ZY, et al.: Use of the UPOINT phenotype system in treating Chinese patients with chronic prostatitis/chronic pelvic pain syndrome: a prospective study. Asian J Androl. 2015, 17:120-3. 10.4103/1008-682X.138189 